\title{
Serum uric acid and high-risk pregnancy: an intriguing correlation in advanced pregnant women
}

\author{
You-Fan Peng ${ }^{1}$, Xiang-Hui Su ${ }^{2}$, Man-Man $\mathrm{Han}^{1}$, Xiang-Yun Zhu ${ }^{1}$, Ling $\mathrm{Li}^{1}$ \\ ${ }^{1}$ Department of Endocrinology, Zhongda Hospital, School of Medicine, Southeast University, Nanjing 210009, China; ${ }^{2}$ Department of \\ Endocrinology, The First Affiliated Hospital of the Medical College, Shihezi University, Shihezi 832003, China \\ Contributions: (I) Conception and design: L Li, YF Peng, (II) Administrative support: L Li; (III) Provision of study materials or patients: L Li; (IV) \\ Collection and assembly of data: MM Han, XY Zhu; (V) Data analysis and interpretation: YF Peng; (VI) Manuscript writing: All authors; (VII) Final \\ approval of manuscript: All authors. \\ Correspondence to: Ling Li. Department of Endocrinology, Zhongda Hospital, School of Medicine, Southeast University, No.87 Dingjiaqiao, Nanjing \\ 210009, China. Email: liling_79@sina.com.
}

\begin{abstract}
Background: The aim of our study is to explore the relationship between serum uric acid (UA) and highrisk pregnancy (HRP) in advanced pregnant women.

Methods: The study included 226 advanced pregnant women ( $\geq 35$ years), and the HRP score were assessed according to China HRP score standards.

Results: All data were separated into the three groups according to HRP score, we observed significant increases of serum UA concentrations between the three groups $(207.51 \pm 42.45 ; 226.65 \pm 45.42$ and $228.27 \pm 49.70 \mu \mathrm{mol} / \mathrm{L}, \mathrm{P}=0.017)$. Notably, serum UA concentrations were found to be positive correlated with HRP score $(\mathrm{r}=0.165, \mathrm{P}=0.013)$ in advanced pregnant women. Serum UA was independent correlated with HRP score (beta $=0.164, \mathrm{P}=0.009$ ) in multiple linear regression analysis.
\end{abstract}

Conclusions: We conclude that serum UA is correlated with HRP score, and increased serum UA levels may herald HRP in advanced pregnant women.

Keywords: Uric acid (UA); high-risk pregnancy (HRP) score; advanced pregnant women

Submitted May 24, 2018. Accepted for publication Oct 08, 2018.

doi: $10.21037 / \mathrm{atm} .2018 .11 .03$

View this article at: http://dx.doi.org/10.21037/atm.2018.11.03

\section{Introduction}

Advanced maternal age is generally defined as pregnancy in women aged 35 years or older. Advanced pregnancy is generally defined as pregnancy in women aged 35 years or older (1). Advanced pregnancy belongs to high-risk pregnancy (HRP) in the clinical management. Pregnant women are considered at high risk if medical conditions may affect maternal or fetal health (2). The national data have indicated that HRP might increase perinatal and neonatal mortality (3). Thus, the assessment of HRP should be considered as a routine examination especially for advanced pregnant woman. In our clinical practice, we have used the HRP score to estimate HRP (4). However, the assessment system is complicated because it approximately includes 84 assessment criteria, and the evaluation procedures may be easily influenced by subjectivity from patients and nonspecialists. So, available and objective laboratory markers are needed to assess the HRP.

Uric acid (UA) has been regarded as an end-product of purine catabolism (5). Recently, serum UA has been reported to be associated with various diseases such as cardiovascular disease, hypertension and metabolic syndrome (6). Previous study attested that serum UA was related with adverse pregnancy outcomes in pregnant woman with chronic glomerulonephritis (7), and serum UA concentrations have been found to be increased in patients with gestational hypertension and preeclampsia $(8,9)$. Obviously, serum UA maintains a close association with pregnancy-related diseases. Thus, the aim of our study is to explore the correlation between serum UA and HRP score 
Table 1 The clinical and laboratory data in advanced pregnant woman

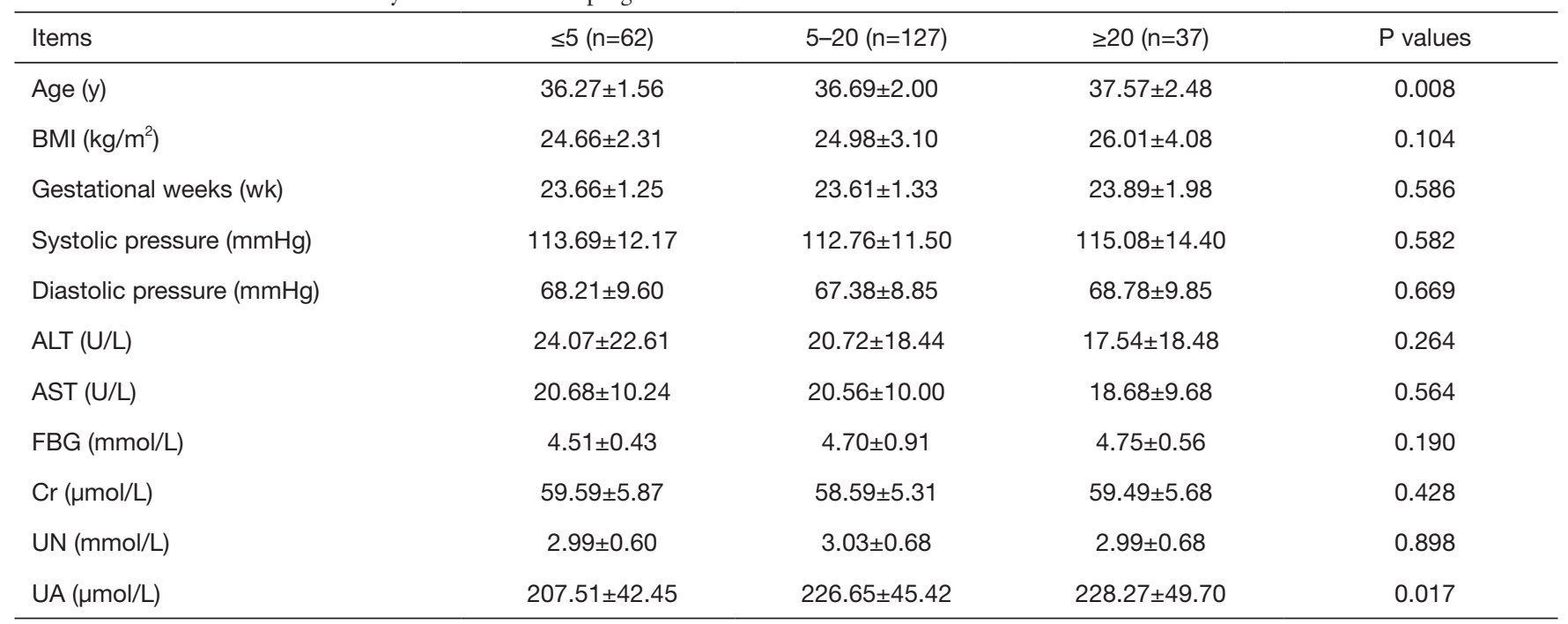

BMI, body mass index; ALT, alanine transaminase; AST, aspartate transaminase; FBG, fasting blood glucose; Cr, creatinine; UN, urea nitrogen; UA, uric acid.

in advanced pregnant women.

\section{Methods}

The study included 226 advanced pregnant women from Zhongda Hospital, School of Medicine, Southeast University (Nanjing, China). Pregnant women older than 35 years were defined as advanced pregnant woman, and pregnancy was diagnosed according to clinical signs, ultrasonic examinations and laboratory examinations. The exclusion criteria included active infection, gout and renal insufficiency.

The study was approved by the Zhongda Hospital, School of Medicine, Southeast University institutional review board (2016ZDSYLL076-P01), and our study was performed according to the guidelines of Helsinki Declaration.

\section{Data collection}

The demographic and laboratory data were collected from the medical records. Fasting blood samples were taken in all participants. All tests were completed in the second trimester of pregnancy in the laboratory, we used the automatic biochemical analyzer (Beckman Coulter, AU 5800, Brea, USA) to measure serum concentrations of fasting blood glucose (FBG), alanine transaminase (ALT), aspartate aminotransferase (AST), creatinine (Cr), urea nitrogen (UN) and UA. The blood pressure was measured by a sphygmomanometer. Body mass index (BMI) was calculated as weight divided by height squared $\left(\mathrm{kg} / \mathrm{m}^{2}\right)$. The HRP score were assessed according to the China high risk pregnancy score standards, which included basic information, pregnancy complication and pregnant disease (4).

\section{Statistical tools}

We used SPSS software version 16.0 to analyze all data. Continuous variables were shown as the means \pm standard deviation. In basic information, the comparisons between the three groups were performed by One-Way ANOVA. We calculated the correlation coefficients between serum UA and other parameters by Pearson and Spearman analysis when appropriate. And we performed multiple linear regression analysis to analyze the independent effects of serum UA levels in all subjects. A P value of $<0.05$ was considered to be statistically significant.

\section{Results}

\section{The clinical and laboratory data in study population}

Table 1 included the characteristics of all subjects, and all data were separated into the three groups according to HRP score. Significant difference in the value distribution of age was found, and we observed significant increases of serum UA concentrations between the three groups (207.51 \pm 42.45 ; $226.65 \pm 45.42$ and $228.27 \pm 49.70 \mu \mathrm{mol} / \mathrm{L}, \mathrm{P}=0.017)$. There were no significant differences for other variables. 


\section{The correlation analyses between serum UA and other parameters in all individuals}

The correlation analyses were carried out in all advanced pregnant women. The positive correlations of serum UA concentrations with BMI, FBG, $\mathrm{Cr}$ and $\mathrm{UN}$ were observed in these pregnant women $(r=0.264, \mathrm{P}<0.001 ; \mathrm{r}=0.136$, $\mathrm{P}=0.041 ; \mathrm{r}=0.243, \mathrm{P}<0.001$ and $\mathrm{r}=0.137, \mathrm{P}=0.040$ ). Notably, serum UA concentrations were found to be positive correlated with HRP score $(\mathrm{r}=0.165, \mathrm{P}=0.013)$ in advanced pregnant women.

\section{The multiple linear regression analysis correlated with serum UA levels}

The basic physiological conditions such as age, blood pressure, gestational weeks and hepatic function might influence serum UA levels $(10,11)$, and serum UA was correlated with BMI, FBG, $\mathrm{Cr}$ and $\mathrm{UN}$ in univariate analysis, therefore, the age, $\mathrm{BMI}$, blood pressure, gestational weeks, FBG, ALT, AST, Cr and UN were included as independent variables in multiple linear regression analysis, and the results found that serum UA was independent correlated with HRP score (beta $=0.164, \mathrm{P}=0.009$ ) in multiple linear regression analysis, as shown in Table 2.

\section{Discussion}

We first found that increased serum UA concentrations showed a correlation with HRP score in advanced pregnant women. Several previous studies have documented the association between serum UA and cardiovascular disease $(12,13)$. Increased serum UA levels have been reported to be associated with hypertension, diabetes mellitus and metabolic syndrome (14-16). On the other hand, elevated serum UA concentrations have been observed in patients with pregnancy-related diseases, such as gestational hypertension, eclampsia and gestational diabetes mellitus $(8,9,17)$.

In our study, we found that serum UA was correlated with HRP score in advanced pregnant women. Traditionally, serum UA has been used as a diagnostic marker for gout and renal injury in clinical laboratories. Recently, a positive correlation between serum UA and C-reactive protein (CRP) in patients with coronary artery ectasia was observed (18). Serum UA as a powerful physiological antioxidant has been reported to be related with acute coronary syndrome, arterial hypertension and cardiovascular disease $(19,20)$. Furthermore, serum UA could predict the alteration of serum CRP concentrations in healthy population (21). In fact, serum UA has antioxidant properties in subjects with abdominal obesity and metabolic syndrome (22), and serum UA concentrations are correlated with biological antioxidant levels in the general population (23), moreover, serum UA is a plasma antioxidant to protect the cells against increased ROS activity (24). Oxidative stress plays an important role in the developments of placental-related diseases, and higher maternal homocysteine as an oxidative stress parameter is associated with risk of abortion or preterm birth in pregnant women (24). Pregnant women with abortion have higher antioxidant enzyme concentrations compared with healthy pregnant women (25). In addition, serum CRP concentrations are correlated with gestational hypertensive complications (26), and oxidative stress increases the risk of gestational complications $(27,28)$. Together, oxidative stress may be a main factor in increasing serum UA concentrations in advanced pregnant women, which may be associated with HRP score in these study subjects, although the in-depth mechanisms remain to be explored.

The present results had some limitations. First, the relationships between serum UA and pregnancy outcomes

Table 2 The factors related with serum UA in advanced pregnant woman in multivariable linear regression analysis

\begin{tabular}{|c|c|c|c|c|c|}
\hline Items & \multicolumn{2}{|c|}{ Unstandardized coefficients } & Standardized coefficients (beta) & $\mathrm{t}$ & $P$ value \\
\hline Age & -3.360 & 1.413 & -0.147 & -2.378 & 0.018 \\
\hline $\mathrm{BMI}$ & 4.281 & 0.915 & 0.290 & 4.681 & $<0.001$ \\
\hline UN & 10.327 & 4.374 & 0.148 & 2.361 & 0.019 \\
\hline HRP score & 1.149 & 0.435 & 0.164 & 2.642 & 0.009 \\
\hline
\end{tabular}

BMI, Body mass index; UN, Urea nitrogen; Cr, Creatinine; HRP, high-risk pregnancy; UA, uric acid. 
have not been investigated. Second, there were no the evaluations for the links between serum UA levels and HRP in pregnant women with normal gestational age ( $<35$ years). Finally, we could not measure the markers of oxidative stress in the present population.

In conclusion, we conclude that serum UA is correlated with HRP score, and increased serum UA levels may herald HRP in advanced pregnant women, however, the relations between serum UA and adverse pregnancy outcomes in advanced pregnant women should be further investigated with a cohort study.

\section{Acknowledgements}

This study was supported by the National Natural Science Foundation of China (No. 81570739).

\section{Footnote}

Conflicts of Interest: The authors have no conflicts of interest to declare.

Ethical Statement: The study was approved by the Zhongda Hospital, School of Medicine, Southeast University institutional review board (2016ZDSYLL076-P01), and our study was performed according to the guidelines of Helsinki Declaration.

\section{References}

1. Laopaiboon $\mathrm{M}$, Lumbiganon $\mathrm{P}$, Intarut $\mathrm{N}$, et al. Advanced maternal age and pregnancy outcomes: a multicountry assessment. BJOG 2014;121 Suppl 1:49-56.

2. Coco L, Giannone TT, Zarbo G. Management of highrisk pregnancy. Minerva Ginecol 2014;66:383-9.

3. Sun L, Yue H, Sun B, et al. Estimation of high risk pregnancy contributing to perinatal morbidity and mortality from a birth population-based regional survey in 2010 in China. BMC Pregnancy Childbirth 2014;14:338.

4. High risk pregnancy score. Available online: https:// wenku.baidu.com/view/976af52cf705cc175427098b.html

5. Choi HK, Mount DB, Reginato AM. Pathogenesis of gout. Ann Intern Med 2005;143:499-516.

6. Borghi C, Rosei EA, Bardin T, et al . Serum uric acid and the risk of cardiovascular and renal disease. J Hypertens 2015;33:1729-41.

7. Sequeira-Alvarado KA, Hernández-Pachecol JA, Espino y Sosa S. First trimester uric acid and adverse pregnancy outcomes in patients with chronic glomerulonephritis during pregnancy. Ginecol Obstet Mex 2015;83:461-6.

8. Yalamati P, Bhongir AV, Betha K, et al. Relationship of serum uric acid, serum creatinine and serum cystatin $\mathrm{C}$ with maternal and fetal outcomes in rural Indian pregnant women. Int J Reprod Contracept Obstet Gynecol 2015;4:1505-10.

9. Corominas AI, Balconi SM, Palermo M, et al . Serum uric acid levels and risk of developing preeclampsia. Medicina (B Aires) 2014;74:462-71.

10. Kawamoto R, Tabara Y, Kohara K, et al. Synergistic influence of age and serum uric acid on blood pressure among community-dwelling Japanese women. Hypertens Res 2013;36:634-8.

11. Zelber-Sagi S, Ben-Assuli O, Rabinowich L, et al.. The association between the serum levels of uric acid and alanine aminotransferase in a population-based cohort. Liver Int2015;35:2408-15.

12. Qin L, Yang Z, Gu H, et al. Association between serum uricacid levels and cardiovasculardisease in middle-aged and elderly Chinese individuals. BMC Cardiovasc Disord 2014;14:26.

13. Juraschek SP, Tunstall-Pedoe H, Woodward M. Serum uric acid and the risk of mortality during 23 years followup in the Scottish Heart Health Extended Cohort Study. Atherosclerosis 2014;233:623-9.

14. Shankar A, Klein R, Klein BE, et al. The association between serum uric acid level and long-term incidence of hypertension: Population-based cohort study. J Hum Hypertens 2006;20:937-45.

15. Dehghan A, Van Hoek M, Sijbrands EJ, et al. High serum uric acid as a novel risk factor for type 2 diabetes. Diabetes Care 2008;31:361-2.

16. Li Q, Yang Z, Lu B, et al. Serum uric acid level and its association with metabolic syndrome and carotid atherosclerosis in patients with type 2 diabetes. Cardiovasc Diabetol 2011;10:72.

17. C R, Samal S, Ghose S. Association of Elevated first Trimester Serum Uric Acid Levels with Development of GDM. J Clin Diagn Res 2014;8:OC01-5.

18. Demir Ş, Karakoyun G,Kanadasi M. Elevated high sensitivity C-reactive protein and uric acid levels in coronary artery ectasia. Acta Biochim Pol 2014;61:687-91.

19. Rodrigo R, Libuy M, Feliú F, et al. Oxidative stressrelated biomarkers in essential hypertension and ischemia-reperfusion myocardial damage. Dis Markers 2013;35:773-90.

20. Spahić E, Hasić S, Kiseljaković E, et al. Positive correlation 
between uric acid and C-reactive protein serum level in healthy individuals and patients with acute coronary syndromes. Med Glas (Zenica) 2015;12:128-32.

21. de Carvalho Vidigal F, de Lima Rosado LE, Paixão Rosado $\mathrm{G}$,et al. Serum uric acid can predict higher C-reactive protein levels in apparently healthy men. Nutr Hosp 2014;29:935-40.

22. Pingmuangkaew $P$, Tangvarasittichai $O$, Tangvarasittichai S. Association of Elevated Serum Uric Acid with the Components of Metabolic Syndrome and Oxidative Stress in Abdominal Obesity Subjects. Indian J Clin Biochem 2015;30:286-92.

23. Ishizaka Y, Yamakado M, Toda A, et al. Relationship between serum uric acid and serum oxidative stress markers in the Japanese general population. Nephron Clin Pract 2014;128:49-56.

24. Micle O, Muresan M, Antal L, et al. The influence of

Cite this article as: Peng YF, Su XH, Han MM, Zhu XY, Li L. Serum uric acid and high-risk pregnancy: an intriguing correlation in advanced pregnant women. Ann Transl Med 2018;6(23):451. doi: 10.21037/atm.2018.11.03 homocysteine and oxidative stress on pregnancy outcome. J Med Life 2012;5:68-73.

25. Baxter N, Sumiya M, Cheng S, et al. Recurrent miscarriage and variant alleles of mannose binding lectin, tumour necrosis factor and lymphotoxin $\alpha$ genes. Clin Exp Immunol 2001;126:529-34.

26. de Jonge LL, Steegers EA, Ernst GD, et al. C-reactive protein levels, blood pressure and the risks of gestational hypertensive complications: the Generation R Study. J Hypertens 2011;29:2413-21.

27. Lekva T, Norwitz ER, Aukrust P, et al. Impact of Systemic Inflammation on the Progression of Gestational Diabetes Mellitus. Curr Diab Rep 2016;16:26.

28. Bartha JL, Fernández-Deudero A, Bugatto F, et al. Inflammation and cardiovascular risk in women with preterm labor. J Womens Health (Larchmt) 2012;21:643-8. 